\title{
A Research on the role of the mass media on Physical Fitness
}

\author{
Ren Xiao-jian ${ }^{1}$, Cui Jiang-ning ${ }^{1}$, Zhang Jin- $\mathrm{i}^{2,}$ a \\ ${ }^{1}$ Shandong Women's University, Shandong Jinan, 250300, China \\ ${ }^{2}$ Key Lab of China's National Linguistic Information Technology, Northwest University for \\ Nationalities, Lanzhou 730030, China \\ aemail: happy0happy@163.com
}

Keywords: the Mass Media; Sports Fitness; the Concept of Sports

\begin{abstract}
The mass media are the most important social information provider, has penetrated into all areas of society. This article starts with the relationship between sports and media, analyzes in depth of the mass media in enhancing people's awareness of fitness, to improves the sports social relations and updates the role of the mass fitness concept, and explores how to improve the mass media to promote its role in promoting the development of sports, puts forward on pay more attention to mass media reports, to broaden the coverage range and channels, increases the format and content of the media reports, enhances the level of reports and other related recommendations.
\end{abstract}

\section{Introduction}

The great success in 2008 Beijing Olympic Games and the 2010 Guangzhou Asian Games in promoting the development of sports undertakings in China are a huge pressure and tests, for China's mass media reported levels and response capacity are also an exploration and a test. To illustrate the nature of sports and the interpretation of the spirit of sport as a global, a state's top sporting events, the Olympic Games and Asian Games provide a good platform for the display, but if there is no the spread of the mass media in this platform, do more efforts will can't achieve the desired results. Because sports display in front of the public the visual image, it is highly dynamic, the audience's attention and excitement mainly focused on the athletes shot of the moment, the moment the sprint, fixed in pumping the ball, dunk action, put it simply, the audience to see sporting events, mainly from the visual and auditory senses, coherent, vivid images and sound of the action and on-site access to the sports competitions excitement and orgasm, in this regard, the mass media of television and Internet occupy a unique advantage, plays the most important role. On the other hand, modern public gradually realized the importance of physical fitness for health from mass media the high-density reports, they recognize that "people" have idle time of physical and mental relaxation,namely the Mass Media and sports fitness.

\section{The Concept and the Social Function of the Mass Media}

\subsection{The Concept of the Mass Media}

The 21st century is the era of rapid development of the media, with the continuous development of science and the raising level of science and technology, the mass media is continuing to make progress, following the newspapers, periodicals, radio, television and the Internet, have appeared in people's lives, the medium of mass media tends to be more diversified, and have more impact on the development of human society.

"Spread" originally derived from the English word "Communication", the meaning is very rich, including "session", "communication", "contacts" "exchange”,” participation" and "traffic", mainly refers to human-to-human point of view, information transferring and feeling exchanging or other related to exchanges. From the perspective of communication studies, mass communication is the particular social group using the advanced communication technologies and means to product and delivery the information and knowledge process. information and knowledge production and delivery process for the majority of the members of the society .In modern society, the mass media of mass communication media, "it's a transfer carrier of large-scale information, it's news 
agencies, newspapers, magazines, books, radio, TV, movies, etc[1], including newspapers, periodicals, television and radio is recognized as the main types of mass media.

\subsection{The Social Function of the Mass Media}

Acquisition and transmission the information, sharing the information resources is the most basic functions of the mass media. The mass media has had to undertake the popularity of social education and heritage social-cultural, the community-oriented education for all, the political, economic, cultural and other aspects of knowledge transmitting to the public. The mass media also assume the functions of the social environment monitoring and supervision by public opinion and continuously push forward the reform and development of the society. Mass media as well as have the leisure and entertainment functions to provide the public as spiritual food. Radio, television, newspapers and magazines, online communication have become the most important and indispensable part in the public daily entertainment life.

\section{The Relationship between the Sport and the Mass Media}

\subsection{Sports Need the Media}

Sports development is an important symbol to promote social development and progress of human civilization, the level of development of sports to some extent reflects a country's comprehensive strength and the degree of social civilization. In order to promote the development of sports in China, improving the level of sports and the physical fitness of the people, promoting the material and spiritual civilization, promoting the harmonious development of society, the 15th meeting of the Standing Committee of the Eighth National People's Congress formally adopted the "Sports Law of the People's Republic of China. "

Sports Law quickly penetrates into the public life in various media reports. The vast land of China formed a national fitness boom, at the same time, the process of the development of sports and sports competitions growing prosperity have increasingly become the focus of attention. Especially in the 2008 Beijing Olympic Games, what shown in front of people is the theme of "Green Olympics, High-tech Olympics and People's Olympics" .In 2010, the Asian Games in Guangzhou in turn "harmonious Asian Games" was shown in front of people, its opening and closing ceremonies, exciting events, the sublime scene of raising of the flag and played the sublime the national anthem after competitions, have given the people of the whole country a very exciting feeling. The spirit of never say die of the athletes in the game; bring the snooker, curling, tennis which civilians did not contact, and other new sports, which all were TV and Internet and other media bring the stimulus and wonderful to life for people.

\subsection{Media Requires Sports}

Until the 29th Olympic Games in 2008 was held, the media rely on the advancement of technology, with the increasingly powerful technological advantages and technological means, the Olympic faith, spirit, ideals, mission and spirit of the transmission around the world, and sent to the hearts of the people from the first Olympic Games of 1896. At the same time, various sports events and projects caused by the unprecedented attention of the people, and get fully popularizing, played a great role in promoting the development of sports. During the 2008 Beijing Olympic Games, the Chinese central set, two sets, five sets, seven sets, five in high-definition cable channels, golf tennis and football situation two pay-per-view channels are used for the Olympic Games live or broadcast, especially in the Guangzhou Asian Games, in the final of the track and field 110 meter, the central set of 13 which is news channels live the grand of get gold medal of Liu Xiang. On January 29, 2011, in the Open tennis game of singles final showdown mosaic Lisite Wales and $\mathrm{Li} \mathrm{Na}$, the central sets of 13 news channels occupied nearly 3 hours to broadcast the game live in prime time which was held in Australia, this shows that the mass media are increasingly inseparable from the sports, the two have been closely linked. Especially in recent years, with the increasingly widespread of mass media, sports media reporters, sports activities and events broadcast time and the number of participation in physical exercise and watching sports events people are doubling.

Table 1: the Statistics of CCTV reporter in the Olympic Games

\begin{tabular}{|l|l|l|l|} 
Year & Country & City & The Number of reporter \\
\hline
\end{tabular}




\begin{tabular}{|c|c|c|c|}
\hline & & & (person) \\
\hline 1984 & USA & Los Angeles & 5 \\
\hline 1988 & Korea & Seoul & 18 \\
\hline 1992 & Spain & Barcelona & 28 \\
\hline 1996 & USA & Atlanta & 59 \\
\hline 2000 & Australia & Sydney & 126 \\
\hline 2004 & Greece & Athens & 160 \\
\hline 2008 & China & Beijing & 3000 \\
\hline
\end{tabular}

Source: CCTV internal documents

Table 2: the Statistics of Cumulative time of CCTV reported the Olympic Games

\begin{tabular}{|c|c|c|c|}
\hline Year & Country & City & Time (Hour) \\
\hline 1984 & USA & Los Angeles & $\leqslant 100$ \\
\hline 1988 & Korea & Seoul & 181 \\
\hline 1992 & Spain & Barcelona & 250 \\
\hline 1996 & USA & Atlanta & 602 \\
\hline 2000 & Australia & Sydney & 584 \\
\hline 2004 & Greece & Athens & 1427 \\
\hline 2008 & China & Beijing & 2796 \\
\hline
\end{tabular}

Source: CCTV internal documents

Table 3: the Statistics of watching Olympic Games by TV set

\begin{tabular}{|c|c|c|c|}
\hline Year & Country & City & Spectators (person) \\
\hline 1996 & USA & Atlanta & 33 \\
\hline 2000 & Australia & Sydney & 36 \\
\hline 2004 & Greece & Athens & 39 \\
\hline 2008 & China & Beijing & 40 \\
\hline
\end{tabular}

Source: Statistics by Nielsen Media Research, Inc.

\section{The Role of Mass Media Played on Physical Fitness}

\subsection{Enhance Public Health Awareness and Participation in Physical Fitness Awareness through the Mass Media}

Dissemination through the mass media, a variety of exciting sporting events, sports coverage, news, people's comments as well as a wide range of sports and leisure entertainment, not only to meet the needs of the public sports recreational, and has played an important role in public health awareness, the cultivation of awareness and sport exercise. For example: snooker, curling, tennis have a strong spectator accepted by civilians the most important thing is through spread by the mass media. Exciting events live or broadcast of the FIFA World Cup, NBA basketball, table tennis tournament, volleyball competitions, not only for the development of sport provides a variety of stage, and more sport being recognized, and accepted by people, a variety of events and their inherent rules of sports were knew by people. Sports has a strong charm and appeal a lot of young people watching sports events in the induction of the mass media, and then physical exercise, to enter into the sports industry.

\subsection{Update the Sports Concept of Public through the Mass Media}

From the 2008 Beijing Olympics Games to the 2010 Guangzhou Asian Games, the mass media sent a large-scale of information and live broadcast signal to every household, sent to every corner of the world. With the media coverage and publicity, the updates and popularity of the sport of Olympic philosophy have a better understanding by people. Olympic spirit emphasizes on participation, emphasizing the "faster, higher, stronger", and building a healthy leisure lifestyle. The dissemination and popularization of these ideas to society play a positive role in promoting as a whole new understanding of sports .During the 29th Beijing Olympic Games, there were 11 consecutive days sports show in Tian-an-men Square, the main display of physical activity is divided into 14 major items, 122 small items, Nearly 20,000 people took part in the sports show performances, there were aerobics dance, broadcast gymnastics, fancy basketball, child care group calisthenics, shuttlecock, soccer and other activities. When the Beijing Health Qigong enthusiasts dressed in uniforms in Tian-an-men Square to participate in the exhibition ,the American television news program use 15-minute to broadcast in United States [2].In 2010 Asian Games in Guangzhou, Guangdong Province promote healthy and harmonious lifestyle of the sun, and actively create a 
very traditional features of mass sports activities brand. In 2009, Guangzhou City, Guangdong Province, launched the "National Fitness Day" activities, continuing to organize the health walking on New Year, the flower of spring Sports, Dragon and Lion jumping Lantern Festival, Across the Pearl River in summer, National Fitness Month, and the health running of thousands , million climbing in autumn, students sunshine sports activities, millions of students sunshine sports activities from the whole country, recess activities, the badminton of "the mayor Cup ", table tennis and so on," Every day activities, every game is unique, every month boutique, there have been the climax", a variety of fitness activities, will become not only activate the general public enthusiasm for the Asian Games, the sublimation of the spirit of the Asian Games boosters. And will become an important carrier of practice the national fitness concept to enhance people's physical, more will be set off the universal welcome Asia, the climax foothold of universal fitness [3].

\section{Improvement Points of Mass Media}

It is now the mass media has made some achievements, but in the spread of sports consciousness, there are still a lot of inadequacies.

As there are strong purposes for the state, the focus of China's sports focus on competitive sports, so ignore the essential requirement of the popularity of the sport, China has formed a "competitive power, sports weak "country phenomenon. This irrational tendency also had a negative impact on the mass media, to ignore the essence and spirit of the sport. Sports news have entertainment in large part, but its also focus is still concentrated in competitive sports, to attract the attention of the audience still by mining competitive sports star scandal and private life, but in fact, the honor should belong to the whole team behind the stars. The mass media should adjust and reform, and gradually focus on the reports of mass sports to promote public understanding of sports, to promote the development of mass sports. With the development of society, the audience of sports coverage increased, and they related to various aspects are growing, The mass media should broaden the scope of the reports to improve the contents and form of reports, in order to adapt to the changing needs of economic development and social structure, while promoting the restore of the social function of sport. For the majority of young people, on the one hand, the sports dissemination of information content should pay more attention to cultivate sports literacy. On the other hand, the mass media should use the sport itself as a medium of communication, by the attention of youth , unearth sports and events which reflecting the spiritual level content, show the social attributes of sport, namely what advocate in Beijing Olympic Games the People's Olympics .

The mass media has gradually mature, and focus on its significant impact on young people, their way of reporting and the reported content in the future is to help young people correctly understand the nature and spirit of the sport, train young people to form a positive attitude towards fitness and physical fitness habits, create an environment which conducive to the healthy growth of young people.

\section{References}

[1] Hu Chun-dong. English teaching methods. Higher Education Press, 1990.

[2] Zhao Hua-yong. The history of the development of China Central Television (1958-2008) [M]. Beijing: China Radio and Television Press, 2008.

[3] Jiang Xin-guo, Li Shi. The harmonious development of 2010 Asian Games in Guangzhou and Guangdong Sports [J]. Huizhou University Learned Journal2010, (3).

[4] Cao Lianzhong, Gao Yuanyuan. Research on the Existing Problems and Countermeasures of the Specialized Talent Training in Our Country's Sports Tourism[J], Journal of Harbin Physical Education Institute. 2006, 24(2), pp.29-30.

[5] Huo Hong, et al. Study on Personnel Training of Sports Tourism in Southwest Region [J], Journal of Chengdu Sport University. 2004, 30(6), pp.26-28. 